\title{
The functional relation between partial discharges and induced charge
}

\author{
Pedersen, Aage; Crichton, George C; McAllister, lain Wilson
}

Published in:

IEEE Transactions on Dielectrics and Electrical Insulation

Link to article, DOI:

$10.1109 / 94.407019$

Publication date:

1995

Document Version

Publisher's PDF, also known as Version of record

Link back to DTU Orbit

Citation (APA):

Pedersen, A., Crichton, G. C., \& McAllister, I. W. (1995). The functional relation between partial discharges and induced charge. IEEE Transactions on Dielectrics and Electrical Insulation, 2(4), 535-543.

https://doi.org/10.1109/94.407019

\section{General rights}

Copyright and moral rights for the publications made accessible in the public portal are retained by the authors and/or other copyright owners and it is a condition of accessing publications that users recognise and abide by the legal requirements associated with these rights.

- Users may download and print one copy of any publication from the public portal for the purpose of private study or research.

- You may not further distribute the material or use it for any profit-making activity or commercial gain

- You may freely distribute the URL identifying the publication in the public portal

If you believe that this document breaches copyright please contact us providing details, and we will remove access to the work immediately and investigate your claim. 


\title{
The Functional Relation between Partial Discharges and Induced Charge
}

\author{
A. Pedersen, G. C. Crichton \\ and I. W. McAllister \\ The Technical University of Denmark, Lyngby, \\ Denmark
}

\begin{abstract}
Analytical expressions are deduced for the quantitative evaluation of the charge induced on an electrode by a partial discharge (PD) within a dielectric body. 'Two approaches have been employed to effect this evaluation. These are characterized by the $\lambda$ function and the $\phi$ function, and the essential relationships to be fulfilled by $\lambda$ and $\phi$ are derived. The principal difference between these functions is how the dielectric polarization is accounted for, either implicitly $(\lambda)$ or explicitly $(\phi)$. This difference is brought clearly into focus. Thereafter the application of these functions to PD studies is considered. It is demonstrated quantitatively that perturbations in the dielectric polarization, brought about by the $\mathrm{PD}$, can contribute significantly to the magnitude of the induced charge. As a consequence, the currently accepted approach to PD energy considerations should be re-examined.
\end{abstract}

\section{INTRODUCTION}

$\mathrm{T}$ HE development of a discharge within a gas-filled void embedded in a dielectric leads to the separation of charge within the void. Almost immediately this space charge brings about the electrification by electrostatic induction of all the conductors of the system. Such a process initially does not involve the transfer of charges either to or from the conductors: see [1] for a full discussion of this phenomenon. With reference to the detecting electrode, this process means the creation of both positive and negative charge distributions on the surface of this electrode. Collectively, these charges are referred to as the induced charge. As discussed previously [1], this induced charge may be identified as being of two distinct components: the Laplacian induced charge and the Poissonian induced charge. The former, which is equal in magnitude but opposite in polarity to the latter, is associated with the change in electrode potential. The latter is directly linked to the void space charge. In the present study we are concerned exclusively with quantify- ing this latter relationship. Consequently, in the ensuing discussions it is frequently convenient to invoke only the common term 'induced charge', but we will imply only the Poissonian component of the induced charge.

The quantitative evaluation of the induced charge can be undertaken with reference to either the $\vec{D}$ field or the $\vec{P}$ field [2], where $\vec{D}$ represents the electric flux density, and $\vec{P}$ denotes the polarization, i.e. the dipole moment density, of the polarized dielectric. For practical applications the former, or Maxwellian, approach is the more relevant. The latter, or quasi-molecular, approach is suitable for fundamental studies in that this approach provides a connection to the molecular aspects of the phenomenon. Although both the Maxwellian and quasimolecular approaches have been discussed previously [2], a more detailed derivation and discussion of these is the initial object of this paper.

Following this, the application of these approaches to $\mathrm{PD}$ analysis is discussed. By separation of the discharge

$1070-9878 / 95 / \$ 3.00$ (C) 1995 IEEE 
phenomenon into two distinct time intervals, a greater insight into the induced-charge transients is made possible. Moreover, contributions to these transients brought about solely by perturbations in the dielectric polarization can be quantified. This deduction has important consequences when the energy associated with the PD itself is under consideration.

\section{BASIC POISSON FIELD}

We consider initially an electrically-stressed dielectric system containing a void. Prior to the occurrence of PD activity, the field associated with the dielectric system will, on account of the applied voltage $U$, be Laplacian. Such a space charge free field can be characterized in terms of the parameters listed in Table 1 , where $\varepsilon$ is the permittivity, $\varepsilon_{0}$ the permittivity of vacuum, and $\vec{r}$ a radius vector. The defining equations for a Laplace field are

$$
\nabla \cdot \vec{D}=0
$$

and

$$
\vec{n} \cdot\left(\vec{D}_{+}-\vec{D}_{-}\right)=0
$$

at the dielectric interfaces. The + and - subscripts refer to the two sides of the interface in question, and $\vec{n}$ is a unit vector directed away from the side which we have selected to call the positive side. We denote the field associated with (1) and (2) the basic Laplace field.

After PD activity, the presence of space charge in the void dictates that the field be Poissonian, and as such the field parameters must reflect this situation, see Table 2, where $\delta V(\vec{r}), \delta \vec{E}(\vec{r}), \delta \vec{D}(\vec{r})$ and $\delta \vec{P}(\vec{r})$ are the field parameters related to the space charges in the void. For this Poisson field we write the associated defining equations as

$$
\nabla \cdot(\vec{D}+\delta \vec{D})=\rho
$$

and

$$
\vec{n} \cdot\left[\left(\vec{D}_{+}+\delta \vec{D}_{+}\right)-\left(\vec{D}_{-}+\delta \vec{D}_{-}\right)\right]=\sigma
$$

at the dielectric interfaces. $\delta \vec{D}$ represents the component of the electric flux density associated with space charge, of volume charge density $\rho$ and surface charge density $\sigma$. The solution of (3) and (4) will be denoted the general Poisson field. However, on the basis of (1) and (2), it is possible to reduce (3) and (4) to

$$
\nabla \cdot \delta \vec{D}=\rho
$$

and

$$
\vec{n} \cdot\left(\delta \vec{D}_{+}-\delta \vec{D}_{-}\right)=\sigma
$$

The solution of (5) and (6) will be defined as the basic Poisson field, and the associated field parameters are also listed in Table 2.

The separation of a general Poisson field into the two components, namely, the basic Laplace field and the basic
Poisson field, implies that, at any instant in time $t$, the total charge $Q_{i}(t)$ on the $i$-th electrode in an $N$ electrode system is related to the Poissonian induced charge $q_{i}(t)$ and the actual voltage $U(t)$, i.e. $U(t)$ includes any voltage transient, by

$$
Q_{i}(t)=q_{i}(t)+\sum_{j=0}^{N} C_{i j}\left[U_{i}(t)-U_{j}(t)\right]
$$

where $C_{i j}$ is the partial capacitance between the $i$-th and $j$-th electrodes and $j=0$ refers to ground. $U_{i}$ and $U_{j}$ are the potentials of the $i$-th and $j$-th electrodes. Although the basis for this relationship may be found in [2], it should be noted that certain parameters are expressed differently in that paper.

\section{MAXWELLIAN APPROACH: THE $\lambda$ FUNCTION}

\subsection{DEFINITION OF THE $\lambda$ FUNCTION}

We consider first the situation of a point charge $d Q$ located in a heterogeneous dielectric system in the presence of electrodes, and that this charge induces charge on all electrodes. From basic electromagnetic theory, it is clear that the charge induced electrostatically on an electrode by space charge depends in a unique way upon the location and magnitude of this space charge. With reference to the $i$-th electrode in an $N$ electrode system, the total Poissonian induced charge $d q_{i}$ related to $d Q$ may be expressed as

$$
d q_{i}=-\lambda_{i} d Q
$$

i.e. $d q_{i}$ also includes the contribution due to changes in the dielectric polarization related to the field of $d Q$. The parameter $\lambda_{i}$ is simply a dimensionless factor of proportionality. In a general system with $N$ electrodes we need $N$ different $\lambda$ functions, one for each electrode. This is necessary as the charge induced by the same source differs from electrode to electrode.

The electrostatically induced charge on the $i$-th electrode associated with a distribution of both volume space charges and charges on dielectric interfaces is, due to the principle of superposition, the sum of the contributions arising from all such space elements $d \Omega$ and surface elements $d S$ of charge, i.e.

$$
q_{i}=-\iiint_{\Omega} \lambda_{i} \rho d \Omega-\iint_{S} \lambda_{i} \sigma d S
$$

The volume charge density $\rho$ and the surface charge density $\sigma$ are functions of location and they may also vary with time, but $\lambda_{i}$ is independent of time. The volume integral is extended to all space $\Omega$ between the electrodes 
Table 1. Electric field parameters after a PD. General and basic Poisson and Laplace fields.

\begin{tabular}{|l|c|c|c|}
\hline \multirow{2}{*}{ Parameter } & Laplace & \multicolumn{2}{|c|}{ Poisson } \\
\cline { 2 - 4 } & Basic & General & Basic \\
\hline applied voltage & $U$ & $U$ & $U=0$ \\
potential distribution & $V(\vec{r})$ & $V(\vec{r})+\delta V(\vec{r})$ & $\delta V(\vec{r})$ \\
electric field strength & $\vec{E}(\vec{r})=-\nabla V(\vec{r})$ & $\vec{E}(\vec{r})+\delta \vec{E}(\vec{r})$ & $\delta \vec{E}(\vec{r})$ \\
electric flux density & $\vec{D}(\vec{r})=\varepsilon \vec{E}(\vec{r})$ & $\vec{D}(\vec{r})+\delta \vec{D}(\vec{r})$ & $\delta \vec{D}(\vec{r})$ \\
dielectric polarization & $\vec{P}(\vec{r})=\vec{D}(\vec{r})-\varepsilon_{0} \vec{E}(\vec{r})$ & $\vec{P}(\vec{r})+\delta \vec{P}(\vec{r})$ & $\delta \vec{P}(\vec{r})$ \\
\hline
\end{tabular}

and the surface integral to all interfaces $S$ between dielectrics.

The $\lambda_{i}$ function has a specific value for every point in space. It takes the value unity at the surface of the $i$-th electrode and tends towards zero as the spatial location recedes from this electrode, and also as it approaches all other electrodes. At the surface of those electrodes its value is zero. Further, provided that the dielectric permittivities are independent of electric field strength, $\lambda_{i}$ is independent of the voltage applied to the system. Equation (9) is the defining equation for $\lambda_{i}$ and as such is used to determine the properties of the $\lambda_{i}$ function. This will now be undertaken.

As a final comment, it should be noted that there is no logical connection between the Poissonian induced charge and the apparent charge as defined in IEC 270 (1981), $\$ 3.2 .2$ [3]. It is nevertheless evident from the actual usage of this latter term that apparent charge is in reality the Poissonian induced charge, see [1].

\subsection{DERIVATION OF THE $\lambda$ FUNCTION}

In order to simplify our derivation of the $\lambda_{i}$ function, we will in our mathematical analysis assume that all $N$ electrodes are held at zero potential, whilst all space charges are fixed in their positions acquired when the voltages were applied to the electrodes. Consequently, as there is then no Laplace field, this implies that, owing to the presence of space charge in the interelectrode volume, the only charges present on the electrodes are the Poissonian induced charges, see (7). In spite of the fact that all electrodes are considered to be grounded, an electric field is nevertheless established throughout the dielectric due to the presence of all such space and surface charges. We can therefore, in our mathematical formulation, replace $\rho$ and $\sigma$ with

$$
\rho=\nabla \cdot \delta \vec{D}
$$

and

$$
\sigma=\vec{n} \cdot\left(\delta \vec{D}_{+}-\delta \vec{D}_{-}\right)
$$

$\delta \vec{D}$ denotes the electric flux density in the basic Poisson field established by the space and surface charges in the dielectric, together with the Poissonian induced charges on the electrodes. We can therefore rewrite $(9)$ in the following manner

$$
q_{i}=-\iiint_{\Omega} \lambda_{i}(\nabla \cdot \delta \vec{D}) d \Omega-\iint_{S} \lambda_{i} \vec{n} \cdot\left(\delta \vec{D}_{+}-\delta \vec{D}_{-}\right) d S
$$

On introducing the vector identity

$$
\nabla \cdot\left(\lambda_{i} \delta \vec{D}\right)=\lambda_{i} \nabla \cdot \delta \vec{D}+\delta \vec{D} \cdot \nabla \lambda_{i}
$$

we obtain

$$
\begin{aligned}
q_{i}= & -\iiint_{\Omega} \nabla \cdot\left(\lambda_{i} \delta \vec{D}\right) d \Omega+\iiint_{\Omega} \delta \vec{D} \cdot \nabla \lambda_{i} d \Omega \\
& -\iint_{S} \vec{n} \cdot\left(\lambda_{i} \delta \vec{D}_{+}-\lambda_{i} \delta \vec{D}_{-}\right) d S
\end{aligned}
$$

For our system, Gauss' generalized theorem $[4,5]$ applied to the vector $\lambda_{i} \delta \vec{D}$ reads

$$
\begin{aligned}
& \iiint_{\Omega} \nabla \cdot\left(\lambda_{i} \delta \vec{D}\right) d \Omega+ \\
& \iint_{S} \vec{n} \cdot\left(\lambda_{i} \delta \vec{D}_{+}-\lambda_{i} \delta \vec{D}_{-}\right) d S+\iint_{F} \vec{c} \cdot\left(\lambda_{i} \delta \vec{D}\right) d F=0
\end{aligned}
$$

where the last surface integral is taken over all electrode surfaces $F$, and $\vec{c}$ is a unit vector outward from the electrode surface. Because $\lambda_{i}=1$ at the $i$-th electrode and $\lambda_{i}=0$ at all other electrodes, this last integral is simply equal to $q_{i}$. A comparison of (14) and (15) shows that it is required that $\lambda_{i}$ be interpreted in a manner such that

$$
\iiint_{\Omega} \delta \vec{D} \cdot \nabla \lambda_{i} d \Omega=0
$$

To obtain a simple condition for $\lambda_{i}$ we introduce the relationship

$$
\delta \vec{D}=\varepsilon \delta \vec{E}=-\varepsilon \nabla \delta V
$$

where $\delta \vec{E}$ is electric field strength and $\delta V$ is the scalar potential in the volume between the electrodes, and $\varepsilon$ is the permittivity. Equation (16) can now be written in the form

$$
\iiint_{\Omega} \nabla \delta V \cdot\left(\varepsilon \nabla \lambda_{i}\right) d \Omega=0
$$


or with reference to the vector identity

$$
\nabla \cdot\left(\delta V \varepsilon \nabla \lambda_{i}\right)=\delta V \nabla \cdot\left(\varepsilon \nabla \lambda_{i}\right)+\nabla \delta V \cdot\left(\varepsilon \nabla \lambda_{i}\right)
$$

in the form

$\iiint_{\Omega} \nabla \cdot\left(\delta V \varepsilon \nabla \lambda_{i}\right) d \Omega-\iiint_{\Omega} \delta V \nabla \cdot\left(\varepsilon \nabla \lambda_{i}\right) d \Omega=0$

We then apply Gauss' generalized theorem to the vector $\delta V \varepsilon \nabla \lambda_{i}$

$$
\begin{aligned}
& \iiint_{\Omega} \nabla \cdot\left(\delta V \varepsilon \nabla \lambda_{i}\right) d \Omega+ \\
& \iint_{S} \vec{n} \cdot\left(\delta V \varepsilon_{+} \nabla_{+} \lambda_{i}-\delta V \varepsilon_{-} \nabla_{-} \lambda_{i}\right) d S+ \\
& \iint_{F} \vec{c} \cdot\left(\delta V \varepsilon \nabla \lambda_{i}\right) d F=0
\end{aligned}
$$

In this last integral $\delta V$ is zero because, in our analysis, the potentials of all electrodes were set to zero. This means that (20) can be written as

$$
\begin{aligned}
& \iiint_{\Omega} \delta V \nabla \cdot\left(\varepsilon \nabla \lambda_{i}\right) d \Omega+ \\
& \iint_{S} \delta V\left[\varepsilon_{+}\left(\frac{\partial \lambda_{i}}{\partial n}\right)_{+}-\varepsilon_{-}\left(\frac{\partial \lambda_{i}}{\partial n}\right)_{-}\right] d S=0
\end{aligned}
$$

It is seen that this condition is fulfilled if

$$
\nabla \cdot\left(\varepsilon \nabla \lambda_{i}\right)=0
$$

and

$$
\varepsilon_{+}\left(\frac{\partial \lambda_{i}}{\partial n}\right)_{+}=\varepsilon_{-}\left(\frac{\partial \lambda_{i}}{\partial n}\right)_{-}
$$

in which $\partial \lambda_{i} / \partial n$ denotes the derivative of $\lambda_{i}$ in the direction normal to the dielectric interface. These conditions, together with the boundary values $\lambda_{i}=1$ at the $i$-th electrode and $\lambda_{i}=0$ at all other electrodes, determine in a unique manner the $\lambda_{i}$ function.

Equation (23) is Laplace's equation, and thus any mathematical method for the calculation of, for example, space charge free electrostatic fields can be employed to evaluate $\lambda_{i}$. It must be emphasized that this does not make $\lambda_{i}$ either a voltage or a potential. Accordingly, $\lambda_{i}$ must not be viewed as a function of the value of the applied voltage.

\section{QUASI-MOLECULAR APPROACH: THE $\phi$ FUNCTION \\ 4.1. MACROSCOPIC REPRESENTATION OF DIELECTRIC POLARIZATION}

In the preceding analysis, the influence of the dielectric polarization upon the magnitude of the induced charge remains concealed in the $\lambda$ function. However, the introduction of a quasi-molecular approach allows us to focus explicitly on the polarization of the dielectric [2] The dielectric polarization $\vec{P}$ is related to the $\vec{D}$ field by

$$
\vec{D}=\varepsilon_{o} \vec{E}+\vec{P}
$$

where $\vec{E}$ is the electric field strength and $\varepsilon_{0}$ the permittivity of vacuum. Hence this approach considers the entire interelectrode volume to be vacuum, in which a distribution of dipoles with a dipole moment density equal to $\vec{P}$ represents the actual dielectric [6-8]. Thus $\vec{P}$ may be related to a point dipole of moment $d \vec{p}$

$$
d \vec{p}=\vec{P} d \Omega
$$

where $d \Omega$ denotes a volume element.

It should be noted that in deriving field solutions which involve dielectric polarization, it is frequently more convenient to replace the distribution of dipoles given by (26) by an equivalent fictitious volume charge density $\rho^{\prime}$, where

$$
\rho^{\prime}=-\nabla \cdot \vec{P}
$$

and an equivalent fictitious surface charge density $\sigma^{\prime}$, where

$$
\sigma^{\prime}=-\vec{n} \cdot\left(\vec{P}_{+}-\vec{P}_{-}\right)
$$

see $[6-10]$ for details. The unit vector $\vec{n}$ is directed away from what we have chosen to denote the positive side of the interface either between two dielectrics, or between polarizable and unpolarizable materials where either $P_{+}$ or $P_{-}$will be zero. Because the two different representations are mathematically equivalent and mutually exclusive, a choice must be made [7-10]. The dipole representation is more convenient for the present study. No fictitious charge is therefore involved in the following discussion.

\subsection{DEFINITION OF THE $\phi$ FUNCTION}

On an electrode, the Poissonian induced charge is now considered to consist of two distinct components, one associated specifically with the space charge distribution, and a second component directly related to the dipoles representing the dielectric. The effective polarization $\vec{P}_{e}$ of a solid dielectric will depend on the applied voltage, and on the field perturbation related to the space charges, i.e.

$$
\vec{P}_{e}=\vec{P}+\delta \vec{P}
$$

where $\vec{P}$ is linked exclusively with the applied voltage, and $\delta \vec{P}$ is associated with the presence of the inter-electrode space charges.

As before, we begin by considering a point charge $d Q$ located in the interelectrode volume. Let $d q_{i Q}$ represent the induced charge resulting from $d Q$ alone. Thus if, 
with reference to an $N$ electrode system, $\phi_{i}$ represents the proportionality factor relating $d q_{i}$ to $d Q$, then we have

$$
d q_{i Q}=-\phi_{i} d Q
$$

In addition, as shown previously [2], the induced charge $d q_{i p}$ on the $i$-th electrode due to a dipole moment $d \vec{p}$ is given by

$$
d q_{i P}=-d \vec{p} \cdot \nabla \phi_{i}
$$

Individually the expressions given in (30) and (31) represent the two components of the induced charge discussed above. Hence, the resulting induced charge on the $i$-th electrode can, with the dipolar representation, be expressed as

$$
q_{i}=-\iiint_{\Omega}\left(\phi_{i} \rho+\delta \vec{P} \cdot \nabla \phi_{i}\right) d \Omega-\iint_{S} \phi_{i} \sigma d S
$$

The volume integral is extended over the entire volume $\Omega$ external to the $N$ electrodes and the surface integral to all dielectric interfaces $S$. It should be noted that the integral expression for $q_{i}$ in (32) is equivalent to that given in (9).

As with the $\lambda_{i}$ function $\phi_{i}$ takes the value unity at the surface of the $i$-th electrode, while its value is zero at all other electrodes. Prior to employing (32), which is the . defining equation for $\phi_{i}$, it is first necessary to establish the required functional basis of $\phi_{i}$ to fulfill (32).

\subsection{DERIVATION OF THE $\phi$ FUNCTION}

As stated previously, we can replace $\rho$ and $\sigma$ with (10) and (11) and hence (32) can be written in the form

$$
\begin{gathered}
q_{i}=-\iiint_{\Omega}\left(\phi_{i} \nabla \cdot \delta \vec{D}+\delta \vec{P} \cdot \nabla \phi_{i}\right) d \Omega- \\
\iint_{S} \vec{n} \cdot\left\{\phi_{i} \delta \vec{D}_{+}-\phi_{i} \delta \vec{D}_{-}\right) d S
\end{gathered}
$$

Note that neither (10), which is one of Maxwell's equations, nor (11) contain any fictitious charge. Introducing the vector identity

$$
\nabla \cdot\left(\phi_{i} \delta \vec{D}\right)=\phi_{i} \nabla \cdot \delta \vec{D}+\delta \vec{D} \cdot \nabla \phi_{i}
$$

(33) can be rewritten in the form

$$
\begin{aligned}
q_{i}= & \iiint_{\Omega}\left[\nabla \cdot\left\langle\phi_{i} \delta \vec{D}\right)-(\delta \vec{D}-\delta \vec{P}) \cdot \nabla \phi_{i}\right] d \Omega- \\
& \iint_{S} \vec{n} \cdot\left(\phi_{i} \delta \vec{D}_{+}-\phi_{i} \delta \vec{D}_{-}\right) d S
\end{aligned}
$$

When applying Gauss' generalized theorem to the vector $\phi_{i} \delta \vec{D}$ we obtain

$$
\begin{gathered}
\iiint_{\Omega} \nabla \cdot\left(\phi_{i} \delta \vec{D}\right) d \Omega+ \\
\iint_{S} \vec{n} \cdot\left(\phi_{i} \delta \vec{D}_{+}-\phi_{i} \delta \vec{D}_{-}\right) d S+ \\
\iint_{F} \vec{c} \cdot\left(\phi_{i} \delta \vec{D}\right) d F=0
\end{gathered}
$$

where the last integral is taken over all electrode surfaces, and $\vec{c}$ is a unit vector outward from each electrode surface. Since $\phi_{i}=1$ at the $i$-th electrode and $\phi_{i}=0$ at all other electrodes the last integral is simply equal to $q_{i}$. Comparing (35) and (36) shows that the functional form of the $\phi_{i}$ function must be such that

$$
\iiint_{\Omega}\left[(\delta \vec{D}-\delta \vec{P}) \cdot \nabla \phi_{i}\right] d \Omega=0
$$

Introducing

$$
\begin{aligned}
& \delta \vec{D}=\varepsilon_{o} \delta \vec{E}+\delta \vec{P} \\
& \delta \vec{E}=-\nabla \delta V
\end{aligned}
$$

into (37) we obtain that the required functional relationship must obey

$$
\iiint_{\Omega} \nabla \delta V \cdot \nabla \phi_{i} d \Omega=0
$$

Applying the vector identity

$$
\nabla \cdot\left(\delta V \nabla \phi_{i}\right)=\delta V \nabla^{2} \phi_{i}+\nabla \delta V \cdot \nabla \phi_{i}
$$

to (39) results in

$\iiint_{\Omega} \nabla \cdot\left(\delta V \nabla \phi_{i}\right) d \Omega-\iiint_{\Omega} \delta V \nabla^{2} \phi_{i} d \Omega=0$

The first volume integral can by means of Gauss' generalized theorem be written in the form

$$
\begin{aligned}
& \iiint_{\Omega} \nabla \cdot\left(\delta V \nabla \phi_{i}\right) d \Omega= \\
& -\iint_{S} \delta V \vec{n} \cdot\left(\nabla_{+} \phi_{i}-\nabla_{-} \phi_{i}\right) d S- \\
& \iint_{F} \delta V \vec{c} \cdot \nabla \phi_{i} d F
\end{aligned}
$$

In the basic Poisson field $\delta V=0$ at all electrode surfaces. Hence the last integral in (42) is zero, and thus (41) can be written in the form

$-\iiint_{\Omega} \delta V \nabla^{2} \phi_{i} d \Omega-\iint_{S} \delta V \vec{n} \cdot\left(\nabla_{+} \phi_{i}-\nabla_{-} \phi_{i}\right) d S=0$ 
In both of these integrals $\delta V$ can attain values which are not zero. Therefore, the functional conditions to be imposed upon the $\phi_{i}$ function are

$$
\nabla^{2} \phi_{i}=0
$$

and

$$
\vec{n} \cdot\left(\nabla_{+} \phi_{i}-\nabla_{-} \phi_{i}\right)=0
$$

As the curl of a gradient is always zero, this implies that the tangential components of $\nabla \phi_{i}$ are the same at the two sides of a dielectric interface. Thus (45) allows us to conclude that no boundary condition is associated with the dielectric interfaces of the system. The sole requirements to be fulfilled are therefore

$$
\nabla^{2} \phi_{i}=0
$$

with $\phi_{i}=1$ at the $i$-th electrode and $\phi_{i}=0$ at all other electrodes.

This is the simplest form of Laplace's equation. It has the same mathematical form as Laplace's equation for a scalar potential in a space charge free electrostatic field in vacuum. However, as stated previously, $\phi_{i}$ itself is a dimensionless quantity.

\section{APPLICATION OF $\lambda$ AND $\phi$ TO PD STUDIES}

Because the detection of PD refers to signals which are manifest at a specific electrode, we will now omit the identifying subscript. In evaluating the induced charge associated with a PD, it is advantageous to consider the event separated into two distinct time intervals;

1. the duration of the void discharge development: $0 \leqslant$ $t \leqslant T_{1}$,

2. the period following the cessation of the discharge development: $T_{1} \leqslant t \leqslant T_{2}$

where $t$ is time. $T_{1}$ denotes the duration of the void discharge development, and $T_{2}$ is the time of occurrence of the next discharge.

In the former time interval, the electrons and ions created in the discharge are in motion within the void. Thus (9) or (32) would have to be employed to determine $q$.

As a result of the applied field, the charges drift to and thereafter accumulate at the surface of the void. The electric field from these surface charges will oppose the applied field within the void. This charge separation process will continue until the discharge is field quenched at time $T_{1}$. If we assume that the wall conductivity $I$ is zero, all surface charges will remain in situ until the occurrence of the next discharge at $T_{2}$. The time interval between the discharge quenching and a succeeding discharge constitutes the latter time interval. In such conditions, $\sigma$ will represent the final value of the surface charge density at the void wall, and thus, with reference to (9) and (32), $q$ is given by either

$$
q=-\iint_{S} \lambda \sigma d S
$$

or

$$
q=-\iiint_{\Omega} \delta \vec{P} \cdot \nabla \phi d \Omega-\iint_{S} \phi \sigma d S
$$

for $T_{1} \leqslant t \leqslant T_{2}$. It should be observed that (47) and (48) represent the final value of $q$ not cnly for a single discharge but also for a series of discharges.
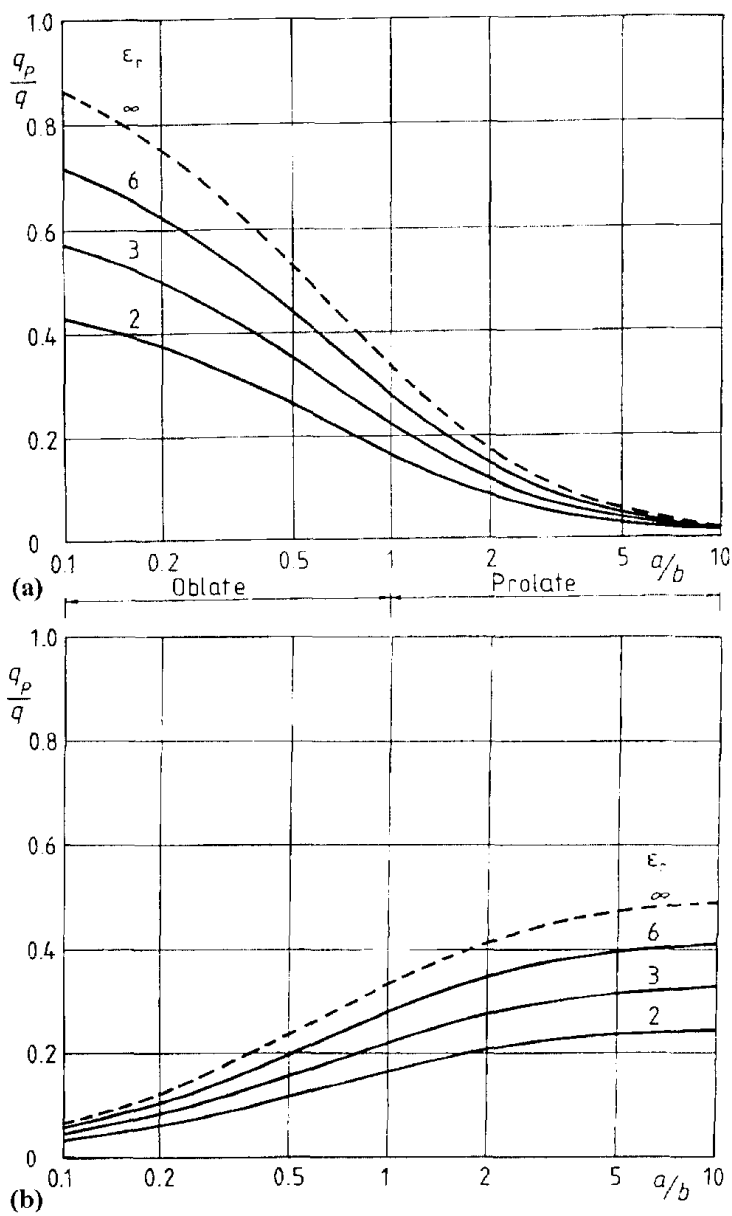

Figure 1.

Variation of the induced charge component associated with changes in dielectric polarization for spheroidal voids. (a) $\nabla \lambda_{0}$ parallel to void axis of rotation. (b) $\nabla \lambda_{0}$ normal to void axis of rotation.

As the void dimensions are normally small relative to those of the system, $\lambda$ and $\phi$ within the void can be replaced by their Taylor expansions. This leads to a 
multipole expansion [11] of the surface integrals in (47) and (48), in which the only significant term is the dipole term, see [2] for details. Hence, with reference to induced charge, the effect of the void surface charges can be considered as the effect of an electric dipole located within the void of dipole moment $\vec{\mu}$, where $\vec{\mu}$ is given by

$$
\vec{\mu}=\iint_{S} \vec{s} \sigma d S
$$

and $\vec{s}$ is the radius vector which locates the position of $d S$ with respect to the point within the void which was chosen as the basis for the Taylor expansions of $\lambda$ and $\phi$. It should be noted that the addition of any constant vector to $\vec{s}$ in (49) does not change the value of the integral. $S$ now represents the surface of the void. This leads to the following expressions for $q$

$$
q=-\vec{\mu} \cdot \nabla \lambda
$$

and

$$
q=-\iiint_{\Omega} \delta \vec{P} \cdot \nabla \phi d \Omega-\vec{\mu} \cdot \nabla \phi
$$

From (50) and (51) it is evident that, through the $\lambda$ and $\phi$ functions, we are able to quantify the contributions to the induced charge which arise either directly from the space charges or from changes in dielectric polarization. To proceed with the analysis, it is appropriate to let

$$
q=q_{P}+q_{\mu}
$$

where $q_{P}$ represents the induced charge associated directly with the change in polarization due to the presence of the void space charge, and $q_{\mu}$ is the induced charge directly associated with this space charge source. By combining (50), (51) and (52) we obtain

$$
q_{P}=-\vec{\mu} \cdot(\nabla \lambda-\nabla \phi)
$$

As we have neglected the second and all higher order terms in the Taylor expansions, this implies that $\nabla \lambda$ is considered to be constant within the void. Consequently, by mathematical analogy with electrostatic fields, we may write [12]

$$
\nabla \lambda=h \nabla \lambda_{0}
$$

where $\lambda_{0}$ represents the $\lambda$ function of the associated voidfree system. For voids of simple form embedded in isotropic dielectrics, the parameter $h$ is a scalar.

For a homogeneous dielectric system, and for a very restricted class of heterogeneous dielectric geometries, $\lambda_{0}$ is a solution of the reduced Laplace equation, and thus we have for such systems

$$
\lambda_{0} \equiv \phi
$$

Hence from (52), (53) and (54) we obtain

$$
q_{P}=-(h-1) \vec{\mu} \cdot \nabla \lambda_{0}
$$

or

$$
\frac{q_{P}}{q}=\frac{h-1}{h}
$$

In a recent publication [12], it was shown that for ellipsoidal voids

$$
h=\frac{K \varepsilon_{r}}{1+(K-1) \varepsilon_{r}}
$$

where $K$ is a dimensionless parameter which arises from an analysis of the electrostatic field of an ellipsoidal void located in an extended uniform field. $\varepsilon_{r}$ is the relative permittivity of the solid dielectric. Introducing (58) into (57) leads to

$$
\frac{q_{P}}{q}=\frac{\varepsilon_{r}-1}{K \varepsilon_{r}}
$$

Alternatively, from (52) and (57), we obtain

$$
\frac{q_{\mu}}{q}=\frac{1}{h}
$$

or

$$
\frac{q_{\mu}}{q}=\frac{1+(K-1) \varepsilon_{r}}{K \varepsilon_{r}}
$$

In the limit $\varepsilon_{r} \rightarrow \infty$, we have

$$
\begin{gathered}
h_{l i m}=\frac{K}{K-1} \\
{\left[\frac{q_{P}}{q}\right]_{l i m}=\frac{1}{K}} \\
{\left[\frac{q_{\mu}}{q}\right]_{l i m}=\frac{K-1}{K}}
\end{gathered}
$$

The variation of $\left(q_{P} / q\right)$ for spheroidal voids is shown in Figure 1 for different $\varepsilon_{r}$ values. If the axis of rotation is of length $2 a$, then for a prolate spheroid we have $a / b \geqslant 1$, while for an oblate spheroid $a / b \leqslant 1$, where $2 b$ represents the length of the minor/major axis of the prolate/oblate spheroid. From Figure 1, it is clear that, depending on the void geometry and orientation relative to $\nabla \lambda_{0}$, changes in the dielectric polarization can account for a significant portion $(>25 \%)$ of the induced charge. When $\nabla \lambda_{0}$ is parallel to the axis of rotation, the largest polarization contribution to the induced charge is obtained with the oblate spheroidal void, see Figure 1(a). In contrast, for $\nabla \lambda_{0}$ normal to the axis of rotation the greatest contribution is provided by the prolate spheroidal void, see Figure $1(b)$. In each case the largest polarization contribution arises when the largest linear dimension of the void lies normal to $\nabla \lambda_{0}$. 


\section{DISCUSSION \\ 6.1. DERIVATION OF THE $\lambda$ AND $\phi$ FUNCTIONS}

Through the phenomenon of electrification by electrostatic induction a partial discharge in a void induces a charge on the detecting electrode. Traditionally, the evaluation of induced charge is undertaken using Green's reciprocal theorem [13-17]. As yet, however, no rigorous proof has been advanced to substantiate this theorem when applied to a heterogeneous system of polarizable materials containing both volume space charges and charged dielectric interfaces; such limitations can be noted in [18] and [19]. Consequently, to provide a definitive evaluation of the induced charge for such general conditions, it becomes necessary to resolve these limitations. To this end we developed, from first principles, an independent approach in which the formulation of reciprocity was not the starting point. Our approach is based on the principle of superposition, Maxwell's equations and vector analysis. In addition we introduce two functions, namely $\lambda$ and $\phi$. These functions differ in the manner by which the dielectric polarization is taken into account. With the $\lambda$ function, dielectric polarization is accounted for implicitly, whereas with the $\phi$ function this phenomenon is dealt with explicitly.

On the basis of their defining equations, i.e. (9) and (32), the nature of the $\lambda$ and $\phi$ functions has been completely established. The former is shown to be a solution of the general Laplace equation for the boundary conditions $\lambda=1$ at the detection electrode and $\lambda=0$ at all other electrodes. The latter function is a solution of the reduced Laplace equation for identical boundary conditions. In the absence of any polarizable material, e.g. in traditional gas discharge studies [20], the $\lambda$ function and $\phi$ function become synonymous.

\subsection{APPLICATION TO PD \\ 6.2.1. THE $\lambda$ AND $\phi$ FUNCTIONS}

The induced charge may be quantified relative to the partial discharge through either the $\lambda$ function or the $\phi$ function. The $\lambda$ function is related to the $\vec{D}$ field and is thus linked directly to the PD space charge. In contrast, the $\phi$ function is related to the $\vec{P}$ field and hence it becomes possible to distinguish between the influence of the PD itself and that of the dielectric polarization upon the induced charge. By this means the importance of changes in polarization $(\delta \vec{P})$ in contributing to the induced charge has been highlighted. Moreover, this effect is shown to be very dependent on both void geometry and the orientation of the void relative to $\nabla \lambda_{0}$.

In practice the direct application of the $\phi$ function is limited in that a knowledge of $\delta \vec{P}$ requires a prior knowledge of the field perturbation $(\delta \vec{E})$ produced by the PD. However, as the source term itself is an unknown quantity, the associated field parameter cannot be determined. Consequently, the quasi-molecular approach has relevance only for analytical studies involving, for example, PD modeling in which the source term is predefined. With the $\vec{D}$ field approach, the influence of the polarization is incorporated in the $\lambda$ function and hence this approach can be readily applied to practical systems.

\subsubsection{INDUCED CHARGE}

With reference to the Poissonian and Laplacian components of the induced charge, a particular point to be emphasized strongly is that such induced charges, determined by means of either the $\lambda$ function or the $\phi$ function, are real charges which exist on the surfaces of the electrodes. They are not in any way image charges, and must not be referred to by that term. Image charges are fictitious charges which one introduces in a specific mathematical procedure, and as such must clearly remain figments of the imagination.

Turning to PD detection practice, it is evident that the so-called apparent charge is in reality the Poissonian induced charge. The latter is an exactly defined quantity, whereas the definition of the former is of an elusive nature. Adoption of the term Poissonian induced charge would increase the precision of the basic terminology.

\subsubsection{ENERGY CONSIDERATIONS}

In the currently accepted philosophy of $\mathrm{PD}$ transients, i.e. when dealt with in terms of an equivalent capacitive circuit, the induced charge (apparent charge) plays a central role in any assessment of the energy dissipated by the discharge within the void $[21,22]$. As shown, however, the Poissonian induced charge consists of two components, one of which is directly related to the space charge within the void, while the other is related to polarization changes external to the void. Consequently, the whole question of the energy dissipated within the void needs to be reformulated from first principles.

\section{CONCLUSION}

$T$ $\mathrm{HE}$ charge induced on a detecting electrode by a $\mathrm{PD}$ can be quantified in terms of either the $\lambda$ function or the $\phi$ function, for which the necessary relationships have been rigorously established. As the $\lambda$ function deals inherently with the presence of polarizable materials, it is the more relevant for practical applications. However, the introduction of the $\phi$ function enables the induced charge contribution associated with changes in the dielectric polarization to be assessed. Depending on the geometry of the void and its orientation relative to $\nabla \lambda_{0}$, this contribution can exceed $50 \%$ of the induced charge. 
The existence of this component of the induced charge implies that the present methods of assessing the energy dissipated within a void following a PD has no valid physical basis.

\section{REFERENCES}

[1] A. Pedersen, G. C. Crichton and I. W. McAllister, "Partial Discharge Detection: Theoretical and Practical Aspects", IEE Proc. - Science, Measurement and Technology, Vol. 142, pp. 29-36, 1995.

[2] A. Pedersen, G. C. Crichton and I. W. McAllister, "The Theory and Measurement of Partial Discharge Transients", IEEE Trans. Elect. Insul., Vol. 26, pp. 487-497, 1991.

[3] IEC Publication 270, Partial Discharge Measurements, 2nd. edition, 1981.

[4] M. Lagally, Vorlesungen über Vektor-Rechnung, Akademische Verlagsgesellschaft, Leipzig, 1928.

[5] G. Joos, Theoretical Physics, Blackie, Glasgow, 1934.

[6] E. Weber, Electromagnetic Fields: Theory and Applications, Wiley, New York, 1950.

[7] J. A. Stratton, Electromagnetic Theory, McGrawHill, New York, 1941.

[8] C. A. Coulson, Electricity, Oliver and Boyd, Edinburgh, 1948.

[9] M. Abraham and R. Becker, The Classical Theory of Electricity and Magnetism, Blackie, Glasgow, 1932.

[10] W. K. H. Panofsky and M. Phillips, Classical Electricity and Magnetism, Addison-Wesley, Reading, 1955.

[11] J. C. Slater and N. H. Frank, Electromagnetism, McGraw-Hill, New York, 1947.

[12] G. C. Crichton, P. W. Karlsson and A. Pedersen, "Partial Discharges in Ellipsoidal and Spheroidal Voids", IEEE Trans. Elect. Insul., Vol. 24, pp. 335$342,1989$.
[13] J. C. Maxwell, A Treatise on Electricity and Magnetism, Vol. I, 1st edition, Clarendon Press, Oxford, 1873 .

[14] J. H. Jeans, The Mathematical Theory of Electricity and Magnetism, Cambridge University Press, Cambridge, 1908.

[15] W. Shockley, "Currents to Conductors Induced by a Moving Point Charge", J. Appl. Phys., Vol. 9, pp. 635-636, 1938.

[16] S. Ramo, "Currents Induced by Electron Motion", Proc. IRE, Vol. 27, pp. 584-585, 1939.

[17] C. H. Gary, "The Relation between Current and Charge Measured at an Electrode and the Density and Velocity of the Surrounding Space Charge" in Breakdown Phenomena of Long Gaps Under Switching Impulse Conditions, IEEE Publication 74 CH0910-0-PWR, pp. 59-61, 1974.

[18] W. R. Smythe, Static and Dynamic Electricity, McGraw-Hill, New York, 1939.

[19] N. Tralli, Classical Electromagnetic Theory, McGraw-Hill, New York, 1963.

[20] A. Pedersen, "On the Electrical Breakdown of Gaseous Dielectrics: An Engineering Approach", IEEE Trans. Elect. Insul., Vol. 24, pp. 721-739, 1989.

[21] R. Bartnikas and E. J. McMahon (eds.), Engineering Dielectrics, Volume I. Corona Measurement and Interpretation, ASTM Special Technical Publication 669, ASTM, Philadelphia, 1979.

[22] F. H. Kreuger, Partial Discharge Detection in High Voltage Equipment, Butterworths, London, 1989.

This paper is based on a presentation given at the 1994 Volt a Colloquium on Partial Discharge Measurements, Como, Italy, 31 August - 2 September 1994.

Manuscript was received on 17 October 1994, in final form $23 \mathrm{June}$ 1995. 